\title{
Accuracy Improvement of the Method of Multiple Scales for Nonlinear Vibration Analyses of Continuous Systems with Quadratic and Cubic Nonlinearities
}

\begin{abstract}
Akira Abe
Department of Information Systems Engineering, Asahikawa National College of Technology, 2-2-1-6 Syunkodai, Asahikawa, Hokkaido 071-8142, Japan

Correspondence should be addressed to Akira Abe, abe@asahikawa-nct.ac.jp

Received 26 August 2010; Revised 18 November 2010; Accepted 22 December 2010

Academic Editor: Cristian Toma

Copyright (C) 2010 Akira Abe. This is an open access article distributed under the Creative Commons Attribution License, which permits unrestricted use, distribution, and reproduction in any medium, provided the original work is properly cited.

This paper proposes an accuracy improvement of the method of multiple scales (MMSs) for nonlinear vibration analyses of continuous systems with quadratic and cubic nonlinearities. As an example, we treat a shallow suspended cable subjected to a harmonic excitation, and investigate the primary resonance of the $m$ th in-plane mode $\left(\Omega \approx \omega_{m}\right)$ in which $\Omega$ and $\omega_{m}$ are the driving and natural frequencies, respectively. The application of Galerkin's procedure to the equation of motion yields nonlinear ordinary differential equations with quadratic and cubic nonlinear terms. The steady-state responses are obtained by using the discretization approach of the MMS in which the definition of the detuning parameter, expressing the relationship between the natural frequency and the driving frequency, is changed in an attempt to improve the accuracy of the solutions. The validity of the solutions is discussed by comparing them with solutions of the direct approach of the MMS and the finite difference method.
\end{abstract}

\section{Introduction}

It is well known that when thin structures are subjected to time variable loads and vibrate with finite amplitudes that are of the order of their thicknesses, nonlinear dynamic behavior occurs frequently. Since the nonlinear behavior cannot be captured through a linear theory of continuous systems, the geometric nonlinear theory is required to properly predict the nonlinear phenomena. A considerable number of publications have studied the vibration problems of nonlinear partial differential equations (e.g., [1-12]). To solve the nonlinear vibration problems of continuous systems, many researchers have used the method of multiple scales (MMSs) developed by Nayfeh and Mook [13] and Nayfeh [14]. Cartmell et al. [15] published an exhaustive literature review on the analyses of weakly nonlinear 
mechanical systems using the MMS, and recently the MMS has been employed to analyze the dynamic characteristics of microelectromechanical systems (MEMSs) (e.g., [16-18]).

For the analyses of nonlinear continuous systems, the application of MMS can be divided into two categories: direct and discretization approaches. In the direct approach, the MMS is applied directly to the governing partial differential equations, whereas, in the discretization approach, the MMS is applied to the ordinary differential equations derived from Galerkin's procedure. By comparing the results obtained from both these approaches in the vibration analysis for the nonlinear continuous systems, the superiority of the direct approach to the discretization approach was reported [19-32].

Abe [33] investigated the nonlinear vibrations of a hinged-hinged Euler-Bernoulli beam resting on a nonlinear elastic foundation with distributed quadratic and cubic nonlinearities by applying Galerkin's procedure and the shooting method [34]. He gave testimony to the fact that this method is more suitable than the direct approach of the MMS. Abe [35] also analyzed the one-to-one internal resonance of a suspended cable possessing quadratic and cubic nonlinearities, and then demonstrated that the solutions obtained from the shooting method in conjunction with Galerkin's procedure were more accurate than those obtained with the direct approach. This means that highly accurate solutions for the vibration analysis of nonlinear continuous systems can be given by properly taking into account the vibration modes in Galerkin's procedure. In the application of the MMS to ordinary differential equations with cubic nonlinearity, it was reported that the accuracy of the solutions was improved by defining a detuning parameter, which expresses the relationship between linear natural frequency and a driving frequency, in the quadratic form [36-40].

This paper presents a remedy for applying the MMS to continuous systems with quadratic and cubic nonlinearities. As an example, we deal with the primary resonance of a shallow suspended cable. First, by employing Galerkin's procedure, the equation of motion is discretized to the ordinary differential equations having quadratic and cubic nonlinear terms. Next, we apply the MMS to the equation and solve the steady-state responses. To improve the accuracy of the solutions obtained with the MMS, the detuning parameter is defined in the quadratic form. Finally, the validity of the analytical results is discussed by comparing these results with those from the direct approach of the MMS and the finite difference method (FDM).

\section{Basic Equations}

Figure 1 shows a shallow suspended cable with a span having a small equilibrium curvature under gravity $g$ in which the initial static configuration $y(x)$ is defined as $y(x)=-4 x(1-x)$. When the cable is subjected to a harmonic vertical load and it vibrates only in the vertical plane (in plane), the nondimensional equation of motion and the boundary conditions for the cable can be expressed as $[30,41]$

$$
\begin{gathered}
\ddot{v}+2 c \dot{v}-v^{\prime \prime}=\alpha\left(v^{\prime \prime}+b y^{\prime \prime}\right) \int_{0}^{1}\left(b y^{\prime} v^{\prime}+\frac{1}{2} v^{\prime 2}\right) d x+f(x) \cos \Omega t \\
v=0 \quad \text { at } x=0,1
\end{gathered}
$$

where $v$ is the nondimensional displacement in the $y$-direction, $c$ is the viscous damping coefficient, and $f(x)$ and $\Omega$ are the distribution and frequency of the load, respectively. The 


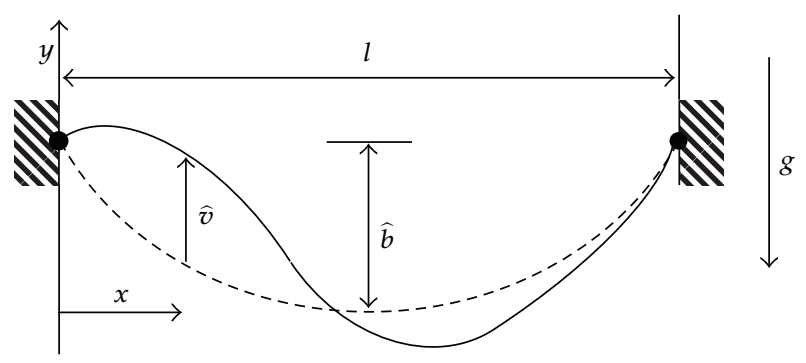

Figure 1: Geometry of a shallow suspended cable.

dot and prime characters express differentiation with respect to the nondimensional time $t$ and coordinate $x$, respectively. The nondimensional parameters are defined as

$$
\alpha=\frac{E A}{H}=\frac{8 b E A}{m g l}, \quad b=\frac{\widehat{b}}{l}
$$

where $E, A$, and $m$ are Young's modulus, the cross-sectional area, and the mass per unit length, respectively.

The displacement function, which satisfies the boundary conditions (2.2), of the cable can be expressed by using the eigenfunction $\phi_{n}$ of the linear vibration as

$$
v(x, t)=\sum_{n=1}^{N} \phi_{n}(x) v_{n}(t)
$$

where

$$
\phi_{n}(x)= \begin{cases}C_{n}\left[1-\cos \left(\omega_{n} x\right)-\tan \left(\frac{\omega_{n}}{2}\right) \sin \left(\omega_{n} x\right)\right] & \text { (for } n=\text { odd) } \\ \sqrt{2} \sin \left(\omega_{n} x\right) & \text { (for } n=\text { even) }\end{cases}
$$

and $v_{n}$ and $\omega_{n}$ are the amplitude and natural frequency of the $n$th mode, respectively. A constant $C_{n}$ is selected such that it satisfies the condition $\int_{0}^{1} \phi_{n}^{2} d x=1$. By substituting (2.4) in (2.1) and performing Galerkin's procedure (i.e., multiplying (2.1) by $\phi_{n}$ and then integrating over the interval $[0,1])$, we obtain ordinary differential equations having quadratic and cubic nonlinear terms

$$
\ddot{v}_{n}+2 c \dot{v}_{n}+\omega_{n}^{2} v_{n}=\sum_{i=1}^{N} \sum_{j=1}^{N} H_{n i j} v_{i} v_{j}+\sum_{i=1}^{N} \sum_{j=1}^{N} \sum_{k=1}^{N} G_{n i j k} v_{i} v_{j} v_{k}+F_{n} \cos (\Omega t)
$$


where $n=1,2,3, \ldots$. The coefficients of the nonlinear terms $H_{n i j}$ and $G_{n i j k}$ are given as

$$
\begin{gathered}
H_{n i j}=\frac{\alpha b}{2} \int_{0}^{1}\left(2 \phi_{i}^{\prime \prime} \int_{0}^{1} y^{\prime} \phi_{j}^{\prime} d x+y^{\prime \prime} \int_{0}^{1} \phi_{i}^{\prime} \phi_{j}^{\prime} d x\right) \phi_{n} d x, \\
G_{n i j k}=\frac{\alpha}{2} \int_{0}^{1}\left(\phi_{i}^{\prime \prime} \int_{0}^{1} \phi_{j}^{\prime} \phi_{k}^{\prime} d x\right) \phi_{n} d x, \quad F_{n}=\int_{0}^{1} f(x) \phi_{n} d x .
\end{gathered}
$$

It should be noted that (2.6) denotes the modal equation of the $n$th vibration mode.

\section{Method of Multiple Scales}

In this section, we derive steady-state solutions for the case of the primary resonance $(\Omega \approx$ $\omega_{m}$ ) by the MMS. First, we introduce a bookkeeping parameter $\varepsilon$, which is later set to 1 , and scale the damping coefficients $c$ and the external load $F_{n}$ to $\varepsilon^{2} c$ and $\varepsilon^{3} F_{n}$, respectively. Next, the amplitude $v_{n}$ is expanded in the form

$$
v_{n}(t)=\varepsilon v_{n 1}\left(T_{0}, T_{1}, T_{2}\right)+\varepsilon^{2} v_{n 2}\left(T_{0}, T_{1}, T_{2}\right)+\varepsilon^{3} v_{n 3}\left(T_{0}, T_{1}, T_{2}\right)+\cdots,
$$

where $T_{p}=\varepsilon^{p} t$ and $p=0,1,2, \ldots$ are different time scales. When the MMS is applied to ordinary differential equations having cubic nonlinear terms, accurate results are given for the primary [36], subharmonic [37], and internal resonances [38-40] by defining the detuning parameter, which expresses the relationship between linear natural frequency and a driving frequency in the quadratic form. We try to improve the accuracy of the MMS for the analysis of ordinary differential equations with quadratic and cubic nonlinearities, and hence the detuning parameter $\sigma$ is redefined from $\Omega=\omega_{m}+\varepsilon^{2} \sigma$ to

$$
\Omega^{2}=\omega_{m}^{2}+\varepsilon^{2} \sigma
$$

By substituting (3.1) and (3.2) in (2.6) and equating the coefficients of $\varepsilon^{1}, \varepsilon^{2}$, and $\varepsilon^{3}$ on both the sides, we obtain

(i) order $\varepsilon^{1}$ :

$$
D_{0}^{2} v_{n 1}+\Lambda_{n}^{2} v_{n 1}=0
$$

(ii) order $\varepsilon^{2}$ :

$$
D_{0}^{2} v_{n 2}+\Lambda_{n}^{2} v_{n 2}=-2 D_{0} D_{1} v_{n 1}+\sum_{i=1}^{N} \sum_{j=1}^{N} H_{n i j} v_{i 1} v_{j 1}
$$


(iii) order $\varepsilon^{3}$ :

$$
\begin{aligned}
D_{0}^{2} v_{n 3}+\Lambda_{n}^{2} v_{n 3}= & -D_{1}^{2} v_{n 1}-2 D_{0} D_{2} v_{n 1}-2 D_{0} D_{1} v_{n 2}-2 c D_{0} v_{n 1} \\
& +\delta_{m n} \sigma v_{n 1}+\sum_{i=1}^{N} \sum_{j=1}^{N} H_{n i j}\left(v_{i 1} v_{j 2}+v_{i 2} v_{j 1}\right) \\
& +\sum_{i=1}^{N} \sum_{j=1}^{N} \sum_{k=1}^{N} G_{n i j k} v_{i 1} v_{j 1} v_{k 1}+F_{n} \cos \left(\Omega T_{0}\right)
\end{aligned}
$$

where $D_{p}=\partial / \partial T_{p}$ and $\delta_{m n}$ is Kronecker's delta. The coefficient $\Lambda_{n}^{2}$ is defined as

$$
\Lambda_{n}^{2}= \begin{cases}\Omega^{2} & (\text { for } n=m), \\ \omega_{n}^{2} & (\text { for } n \neq m) .\end{cases}
$$

When there are no internal resonances among the vibration modes, the general solutions of (3.3) can be given by

$$
\begin{gathered}
v_{m 1}=A_{m}\left(T_{1}, T_{2}\right) e^{i \Omega T_{0}}+c c, \\
v_{n 1}=0, \quad \forall n \neq m,
\end{gathered}
$$

where $c c$ indicates the complex conjugate of the preceding term. By substituting (3.7) in (3.4) and eliminating the secular terms from $v_{n 2}$ (i.e., $D_{1} A_{m}=0$ ), the solution of the order $\varepsilon^{2}$ can be obtained as

$$
v_{n 2}=L_{1 n}\left(A_{m}^{2} e^{2 i \Omega T_{0}}+c c\right)+L_{2 n} A_{m} \bar{A}_{m}
$$

where

$$
L_{1 n}=\frac{H_{n m m}}{\Lambda_{n}^{2}-4 \Omega^{2}}, \quad L_{2 n}=\frac{2 H_{n m m}}{\Lambda_{n}^{2}}
$$

and $\bar{A}_{m}$ is a complex conjugate of $A_{m}$. Next, the substitution of (3.7) and (3.8) in (3.5) yields the solvability condition in the form

$$
2 i \Omega\left(D_{2} A_{m}+c A_{m}\right)+\Gamma_{m} A_{m}^{2} \bar{A}_{m}-\sigma A_{m}=\frac{F_{m}}{2},
$$

where

$$
\Gamma_{m}=-3 G_{m m m m}-\sum_{i=1}^{N}\left(H_{m m i}+H_{m i m}\right)\left(L_{1 i}+L_{2 i}\right) .
$$


To solve (3.10), we express $A_{m}$ and $\bar{A}_{m}$ as

$$
A_{m}=\frac{a}{2} \exp (i \beta), \quad \bar{A}_{m}=\frac{a}{2} \exp (-i \beta)
$$

If we split (3.10) into real and imaginary parts by means of (3.12), the following equations are obtained:

$$
\begin{gathered}
\Omega\left(D_{2} a+c a\right)=-\frac{F_{m}}{2} \sin \beta \\
-\Omega a D_{2} \beta+\left(\frac{\Gamma_{m} a^{2}}{4}-\sigma\right) \frac{a}{2}=\frac{F_{m}}{2} \cos \beta .
\end{gathered}
$$

By substituting conditions $D_{2} a=D_{2} \beta=0$ for the steady-state solutions in (3.13), algebraic equations in terms of unknown variables $a$ and $\beta$ are derived. The steady-state solutions can be obtained from the algebraic equations, and then the in-plane deflection of the cable is given by

$$
v(x, t)=\varepsilon \phi_{m}(x) a \cos (\Omega t+\beta)+\frac{1}{2} \varepsilon^{2} a^{2} \sum_{i=1}^{N}\left\{\phi_{i}(x)\left[L_{1 i} \cos \left(2 \Omega t+2 \beta_{m}\right)+\frac{1}{2} L_{2 i}\right]\right\} .
$$

\section{Finite Difference Method}

The application of analytical approaches to nonlinear continuous systems may lead to unreal results (e.g., [38]), and hence we should check the validity of the analytical solutions. In this paper, the accuracy of analytical results is confirmed by comparing our results with those obtained by the FDM (i.e., direct numerical integration).

The suspended cable is first divided into I short segments separated by an incremental distance $\xi$ and the time is divided into a series of steps of duration $\tau$. If the time and spatial difference operators in (2.1) are approximated by means of central difference derivatives

$$
\frac{\partial(\cdot)}{\partial s} \approx \frac{(\cdot)_{k+1}-(\cdot)_{k-1}}{2 \Delta s}, \quad \frac{\partial^{2}(\cdot)}{\partial s^{2}} \approx \frac{(\cdot)_{k+1}-2(\cdot)_{k}+(\cdot)_{k-1}}{(\Delta s)^{2}}
$$

then (2.1) can be discretized as

$$
U_{i, j+1}=\frac{\tau^{2} Q+(c \tau-1) U_{i, j-1}+2 U_{i, j}}{1+c \tau}
$$


where

$$
\begin{gathered}
U_{i, j}=v(i \xi, j \tau)=v(x, t), \\
Q=U_{D 2}+\alpha\left(U_{D 2}+8 b\right) S+f(i \xi) \cos (\Omega j \tau), \\
U_{D 2}=\frac{U_{i+1, j}-2 U_{i, j}+U_{i-1, j}}{\xi^{2}}
\end{gathered}
$$

and the subscripts $i$ and $j$ indicate the spatial node $(i=0,1,2, \ldots, I)$ and the time step $(j=$ $0,1,2, \ldots, J)$, respectively. The variable $S$ denotes the definite integral in (2.1)

$$
\int_{0}^{1}\left(b y^{\prime} v^{\prime}+\frac{1}{2} v^{\prime 2}\right) d x
$$

By using Simpson's formula, the value is calculated from

$$
S=\left(s_{0}+2 \sum_{i=1}^{I / 2-1} s_{2 i}+2 \sum_{i=1}^{I / 2} s_{2 i-1}+s_{I}\right) \frac{\tau}{3}
$$

where

$$
\begin{gathered}
s_{i}=b[8(i \xi)-4] U_{D 1}+\frac{\left(U_{D 1}\right)^{2}}{2}, \\
U_{D 1}=\frac{\left(U_{i+1, j}-U_{i-1, j}\right)}{2 \xi} .
\end{gathered}
$$

In this study, the parameters are taken to be $I=200$ and $\tau=(2 \pi / \Omega) / 10000$.

\section{Numerical Results and Discussion}

In this section, we examine the primary resonance of the first antisymmetric mode (the second in-plane mode). The following simulation results are provided to demonstrate the effectiveness of the proposed approach presented in Section 3, in which the analytical results are compared with those obtained from the direct approach of the MMS [30] and the FDM presented in Section 4. It should be noted that the detuning parameter $\sigma$ is defined as $\Omega=\omega_{2}+\varepsilon^{2} \sigma$ in the direct approach. The values of the parameters $\alpha$ and $c$ are set to be $\alpha=239.16$ and $c=0.02$, respectively. It is assumed that only the first antisymmetric mode is directly excited by the load. Hence, the distribution $f(x)$ is defined as $f(x)=\bar{F} \phi_{2}(x)$ and the amplitude of the load is taken as $\bar{F}=0.001$. Since the value of the parameter $\alpha b^{2}$ has a significant effect on the nonlinear dynamic characteristics (i.e., hardening or softening type) [30], we also change the value of $\alpha b^{2}$ in the numerical examples.

In the analysis of the MMS presented in Section 3, the accuracy of numerical results is dependent upon the number of terms (2.4). We hence examine first of all convergence characteristics of steady-state responses as the number of terms $N$ of (2.4) increases. 


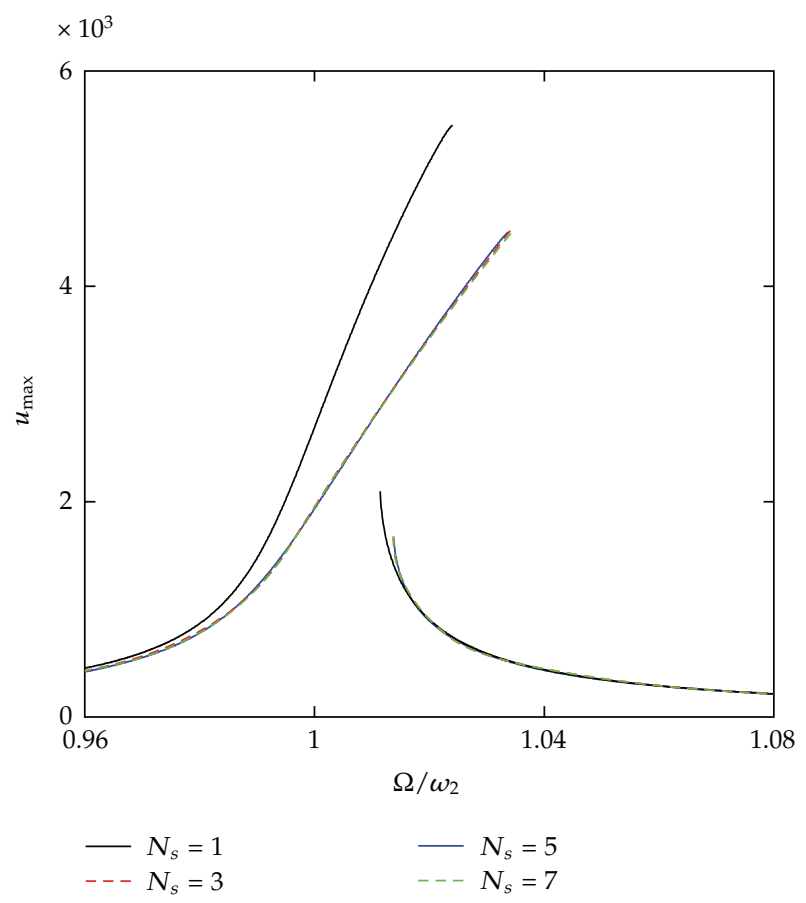

Figure 2: Convergence characteristics of frequency-response curves for the cable $\left(\alpha b^{2}=2\right)$.

Figure 2 shows a convergence study for the stable response of the cable $\left(\alpha b^{2}=2\right)$ when a driving frequency $\Omega$ is near $\omega_{2}$ (i.e., natural frequency of the first antisymmetric mode). The ordinate $u_{\max }$ represents the maximum displacement of the cable at $x=0.25$. The number of the symmetric mode considered in the analysis is denoted by $N_{s}$. It can be seen from Figure 2 that the responses converge with an increase in number of the terms. Therefore, we consider the first to fifth symmetric modes (i.e., $N_{s}=5$ ) in addition to the first antisymmetric mode in the analysis of the MMS.

Figure 3 shows the frequency-response curves near a primary resonance $\left(\Omega \approx \omega_{2}\right)$ in which only the stable responses are plotted. Figures $3(a), 3(b), 3(c)$, and $3(d)$ present the results for the values $\alpha b^{2}=1,2,3$, and 4, respectively. Solid, broken, and dotted lines indicate the responses obtained by using the discretization approach of the MMS mentioned in Section 3, the direct approach of the MMS [30], and the FDM, respectively. In the FDM, the calculation is performed for 10,000 periods so that the transient response decays. It can be observed in all these cases that the results obtained by the present approach approximately coincide with those obtained by the FDM. On the other hand, the amplitudes predicted by the direct approach are smaller than those predicted by the present approach and the FDM. This tendency appears remarkably in Figures 3(b) and 3(c). Therefore, it can be said that the soft (or hard) spring characteristic is prone to appear when using the direct approach of the MMS.

As an example, the normalized spatial distribution of the displacement $v(x)$ for the cable $\left(\alpha b^{2}=2\right.$, and $\left.\Omega / \omega_{2}=1.025\right)$ shown in Figure $3(b)$ is illustrated in Figure 4 . The results of the FDM are denoted by the symbol o. As seen in Figure 4, the results of the present approach are in good agreement with those of the FDM, whereas those of the direct approach are distinctly different near $x=0.75$. Therefore, we confirm that the present approach is valid and is more accurate than the direct approach. 


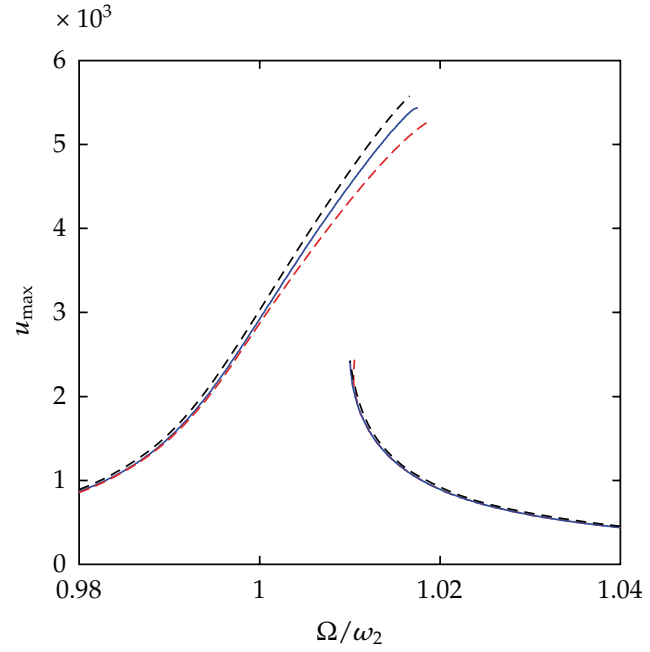

(a)

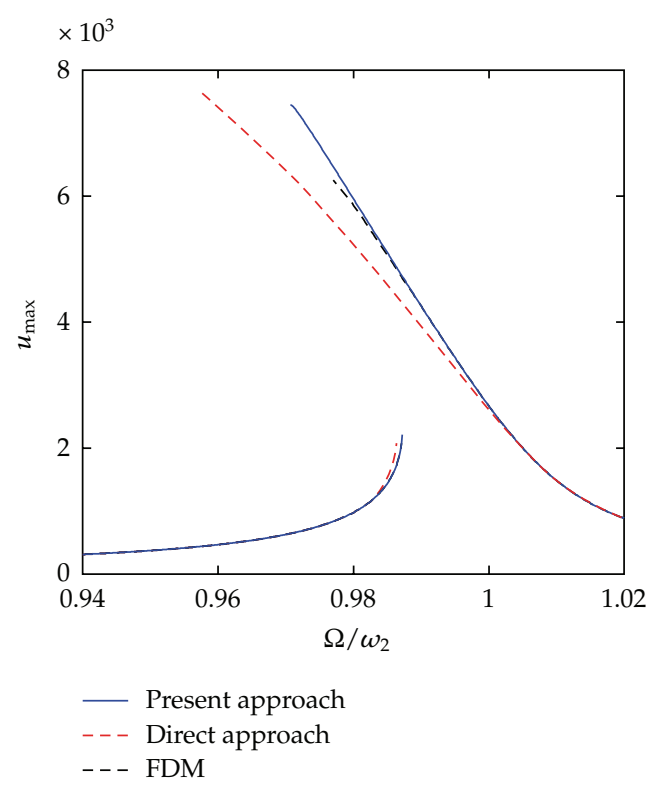

(c)

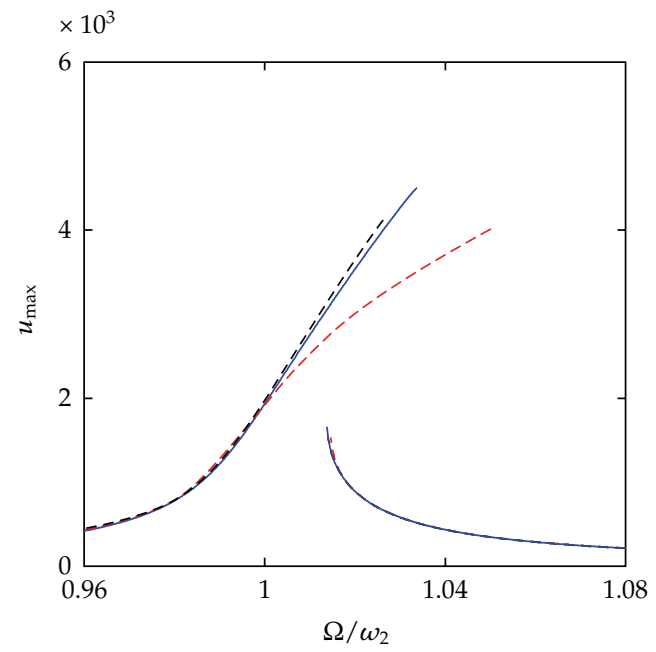

(b)

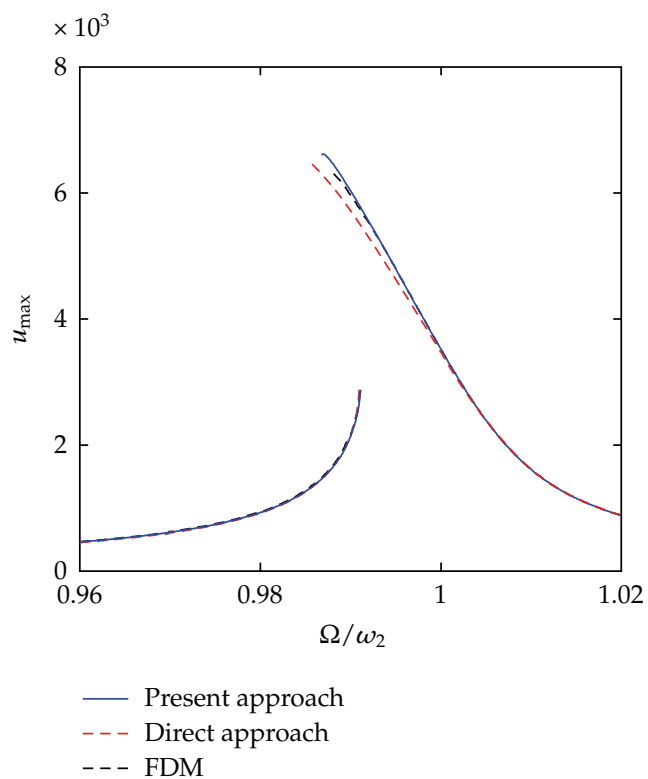

(d)

Figure 3: Comparison of three approaches on the stable response: (a) $\alpha b^{2}=1$, (b) $\alpha b^{2}=2$, (c) $\alpha b^{2}=3$, and (d) $\alpha b^{2}=4$.

In a discretization approach of the MMS using the conventional detuning parameter $\Omega=\omega_{m}+\varepsilon^{2} \sigma$, the solutions for a continuous system with quadratic and cubic nonlinearities become equal to those of the direct approach of the MMS when an infinite number of vibration modes are used in the discretization process [21,30]. However, as mentioned above, the present discretization approach of the MMS in which a finite number of the vibration modes are taken into account is superior to the direct approach in regard to the accuracy of the solutions. Therefore, we conclude that by redefining the detuning parameter 


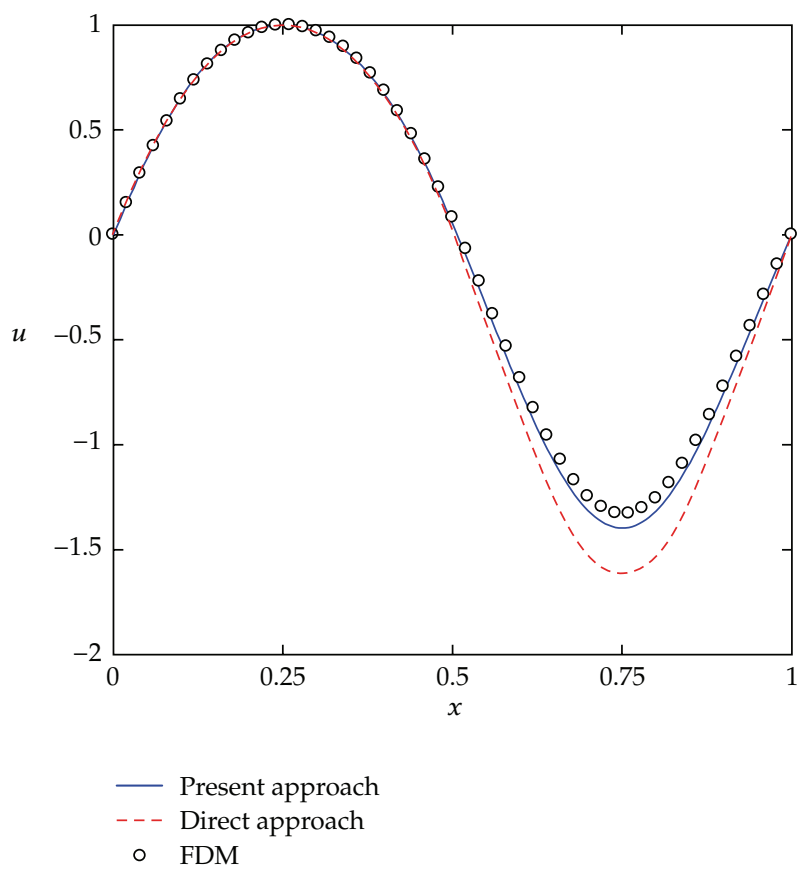

Figure 4: Comparison of three approaches on the normalized spatial distribution of the displacement $v(x)\left(\alpha b^{2}=2, \Omega / \omega_{2}=1.025\right)$.

from $\Omega=\omega_{m}+\varepsilon^{2} \sigma$ to $\Omega^{2}=\omega_{m}^{2}+\varepsilon^{2} \sigma$, the accuracy of the solutions predicted by the discretization approach of the MMS is improved for continuous systems with quadratic and cubic nonlinearities.

\section{Concluding Remarks}

Nonlinear vibrations of a suspended cable with quadratic and cubic nonlinearities were investigated using the discretization approach of the MMS, where we focused on the primary resonance of the first antisymmetric mode (the second in-plane mode). To accurately obtain the solutions, we defined a detuning parameter, which expresses the relationship between linear natural frequency and a driving frequency, in the quadratic form. By comparing the results predicted by the direct approach of the MMS and the FDM, we demonstrated that the present approach is valid and is more suitable than the direct approach.

Many researchers have investigated the dynamic characteristics of continuous systems with quadratic and cubic nonlinearities by using the direct approach. However, since the direct approach cannot adopt the detuning parameter defined in the quadratic form, highly accurate solutions may not be obtained. Hence, the validity of the solutions predicted by the direct approach of the MMS for nonlinear continuous systems should be checked in comparison to those predicted by other approaches.

\section{Acknowledgment}

The author gratefully acknowledges the helpful comments and suggestions of the reviewers, which have improved the presentation. 


\section{References}

[1] J. B. Keller and S. Kogelman, "Asymptotic solutions of initial value problems for nonlinear partial differential equations," SIAM Journal on Applied Mathematics, vol. 18, pp. 748-758, 1970.

[2] S. C. Chikwendu and J. Kevorkian, "A perturbation method for hyperbolic equations with small nonlinearities," SIAM Journal on Applied Mathematics, vol. 22, pp. 235-258, 1972.

[3] P. L. Chow, "Asymptotic solutions of inhomogeneous initial boundary value problems for weakly nonlinear partial differential equations," SIAM Journal on Applied Mathematics, vol. 22, no. 4, pp. 629647, 1972.

[4] R. W. Lardner, "Asymptotic solutions of nonlinear wave equations using the methods of averaging and two-timing," Quarterly of Applied Mathematics, vol. 35, no. 2, pp. 225-238, 1977/78.

[5] A. C. J. Stroucken and F. Verhulst, "The Galerkin-averaging method for nonlinear, undamped continuous systems," Mathematical Methods in the Applied Sciences, vol. 9, no. 4, pp. 520-549, 1987.

[6] W. T. van Horssen, "Asymptotic theory for a class of initial-boundary value problems for weakly nonlinear wave equations with an application to a model of the galloping oscillations of overhead transmission lines," SIAM Journal on Applied Mathematics, vol. 48, no. 6, pp. 1227-1243, 1988.

[7] M. S. Krol, "On a Galerkin-averaging method for weakly nonlinear wave equations," Mathematical Methods in the Applied Sciences, vol. 11, no. 5, pp. 649-664, 1989.

[8] A. C. Lazer and P. J. Mckenna, "Large-amplitude periodic oscillations in suspension bridges. Some new connections with nonlinear analysis," SIAM Review, vol. 32, no. 4, pp. 537-578, 1990.

[9] W. T. V. Horssen, "Asymptotics for a class of semilinear hyperbolic equations with an application to a problem with a quadratic nonlinearity," Nonlinear Analysis, vol. 19, no. 6, pp. 501-530, 1992.

[10] G. J. Boertjens and W. T. Van Horssen, "On mode interactions for a weakly nonlinear beam equation," Nonlinear Dynamics, vol. 17, no. 1, pp. 23-40, 1998.

[11] G. J. Boertjens and W. T. Van Horssen, "Asymptotic theory for a weakly nonlinear beam equation with a quadratic perturbation," SIAM Journal on Applied Mathematics, vol. 60, no. 2, pp. 602-632, 2000

[12] G. J. Boertjens and W. T. Van Horssen, "On interactions of oscillation modes for a weakly non-linear undamped elastic beam with an external force," Journal of Sound and Vibration, vol. 235, no. 2, pp. 201-217, 2000.

[13] A. H. Nayfeh and D. T. Mook, Nonlinear Oscillations, Wiley-Interscience, New York, NY, USA, 1979.

[14] A. H. Nayfeh, Introduction to Perturbation Techniques, Wiley-Interscience, New York, NY, USA, 1981.

[15] M. P. Cartmell, S. W. Ziegler, R. Khanin, and D. I. M. Forehand, "Multiple scales analyses of the dynamics of weakly nonlinear mechanical systems," Applied Mechanics Reviews, vol. 56, no. 5, pp. 455-491, 2003.

[16] M. F. Daqaq, E. M. Abdel-Rahman, and A. H. Nayfeh, "Two-to-one internal resonance in microscanners," Nonlinear Dynamics, vol. 57, no. 1-2, pp. 231-251, 2009.

[17] S. Gutschmidt and O. Gottlieb, "Bifurcations and loss of orbital stability in nonlinear viscoelastic beam arrays subject to parametric actuation," Journal of Sound and Vibration, vol. 329, no. 18, pp. 3835-3855, 2010.

[18] H. M. Ouakad and M. I. Younis, "The dynamic behavior of MEMS arch resonators actuated electrically," International Journal of Non-Linear Mechanics, vol. 45, no. 7, pp. 704-713, 2010.

[19] A. H. Nayfeh, J. F. Nayfeh, and D. T. Mook, “On methods for continuous systems with quadratic and cubic nonlinearities," Nonlinear Dynamics, vol. 3, no. 2, pp. 145-162, 1992.

[20] M. Pakdemirli, "A comparison of two perturbation methods for vibrations of systems with quadratic and cubic nonlinearities," Mechanics Research Communications, vol. 21, no. 2, pp. 203-208, 1994.

[21] M. Pakdemirli and H. Boyaci, "Comparison of direct-perturbation methods with discretizationperturbation methods for non-linear vibrations," Journal of Sound and Vibration, vol. 186, no. 5, pp. 837-845, 1995.

[22] M. Pakdemirli, S. A. Nayfeh, and A. H. Nayfeh, "Analysis of one-to-one autoparametric resonances in cables-Discretization vs. direct treatment," Nonlinear Dynamics, vol. 8, no. 1, pp. 65-83, 1995.

[23] C. M. Chin and A. H. Nayfeh, "Bifurcation and chaos in externally excited circular cylindrical shells," ASME Journal of Applied Mechanics, vol. 63, no. 3, pp. 565-574, 1996.

[24] A. H. Nayfeh and W. Lacarbonara, "On the discretization of distributed-parameter systems with quadratic and cubic nonlinearities," Nonlinear Dynamics, vol. 13, no. 3, pp. 203-220, 1997.

[25] A. Nayfeh, "Reduced-order models of weakly nonlinear spatially continuous systems," Nonlinear Dynamics, vol. 16, no. 2, pp. 105-125, 1998. 
[26] W. Lacarbonara, A. Nayfeh, and W. Kreider, "Experimental validation of reduction methods for nonlinear vibrations of distributed-parameter systems: analysis of a buckled beam," Nonlinear Dynamics, vol. 17, no. 2, pp. 95-117, 1998.

[27] A. H. Nayfeh and W. Lacarbonara, "On the discretization of spatially continuous systems with quadratic and cubic nonlinearities," JSME International Journal C, vol. 41, no. 3, pp. 510-531, 1998.

[28] G. Rega, W. Lacarbonara, A. H. Nayfeh, and C. M. Chin, “Multiple resonances in suspended cables: direct versus reduced-order models," International Journal of Non-Linear Mechanics, vol. 34, no. 5, pp. 901-924, 1999.

[29] W. Lacarbonara, "Direct treatment and discretizations of non-linear spatially continuous systems," Journal of Sound and Vibration, vol. 221, no. 5, pp. 849-866, 1999.

[30] H. N. Arafat and A. H. Nayfeh, "Non-linear responses of suspended cables to primary resonance excitations," Journal of Sound and Vibration, vol. 266, no. 2, pp. 325-354, 2003.

[31] W. Lacarbonara, G. Rega, and A. H. Nayfeh, "Resonant non-linear normal modes. Part I: analytical treatment for structural one-dimensional systems," International Journal of Non-Linear Mechanics, vol. 38, no. 6, pp. 851-872, 2003.

[32] A. F. El-Bassiouny, "On methods for continuous systems with quadratic, cubic and quantic nonlinearities," Chaos, Solitons and Fractals, vol. 39, no. 3, pp. 1308-1316, 2009.

[33] A. Abe, "On non-linear vibration analyses of continuous systems with quadratic and cubic nonlinearities," International Journal of Non-Linear Mechanics, vol. 41, no. 8, pp. 873-879, 2006.

[34] H. Tamura and K. I. Matsuzaki, "Numerical scheme and program for the solution and stability analysis of a steady periodic vibration problem," JSME International Journal C, vol. 39, no. 3, pp. 456463, 1996.

[35] A. Abe, "Validity and accuracy of solutions for nonlinear vibration analyses of suspended cables with one-to-one internal resonance," Nonlinear Analysis: Real World Applications, vol. 11, no. 4, pp. 2594-2602, 2010.

[36] T. Kondou and K. Yagasaki, "Some recent topics on nonlinear vibration and chaos," Transactions of the Japan Society of Mechanical Engineers C, vol. 61, no. 583, pp. 746-451, 1995 (Japanese).

[37] A. Abe, Y. Kobayashi, and G. Yamada, "Analysis of subharmonic resonance of moderately thick antisymmetric angle-ply laminated plates by using method of multiple scales," Journal of Sound and Vibration, vol. 217, no. 3, pp. 467-484, 1998.

[38] A. Abe, Y. Kobayashi, and G. Yamada, "Two-mode response of simply supported, rectangular laminated plates," International Journal of Non-Linear Mechanics, vol. 33, no. 4, pp. 675-690, 1998.

[39] A. Abe, Y. Kobayashi, and G. Yamada, "Three-mode response of simply supported, rectangular laminated plates," JSME International Journal C, vol. 41, no. 1, pp. 51-59, 1998.

[40] A. Abe, Y. Kobayashi, and G. Yamada, "Internal resonance of rectangular laminated plates with degenerate modes," JSME International Journal C, vol. 41, no. 4, pp. 718-726, 1998.

[41] A. H. Nayfeh, H. N. Arafat, C. M. Chin, and W. Lacarbonara, "Multimode interactions in suspended cables," Journal of Vibration and Control, vol. 8, no. 3, pp. 337-387, 2002. 


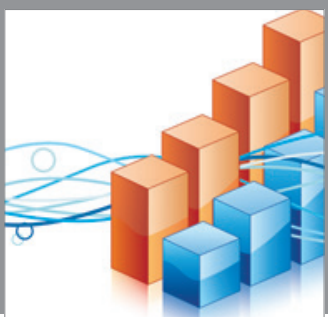

Advances in

Operations Research

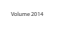

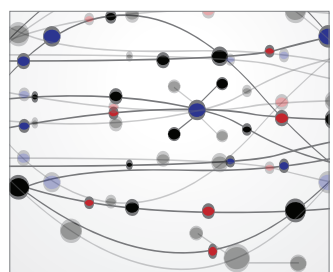

\section{The Scientific} World Journal
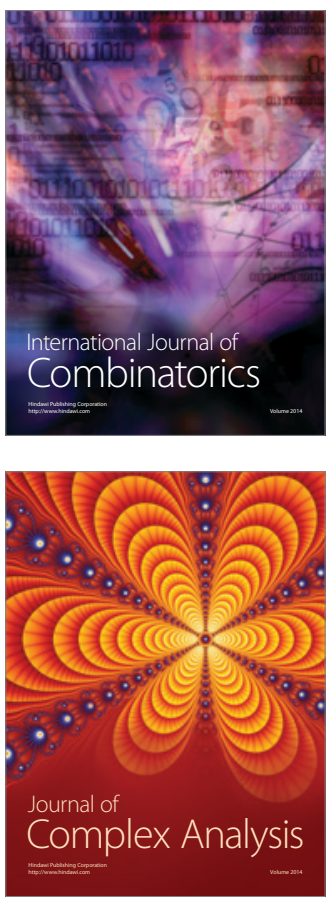

International Journal of

Mathematics and

Mathematical

Sciences
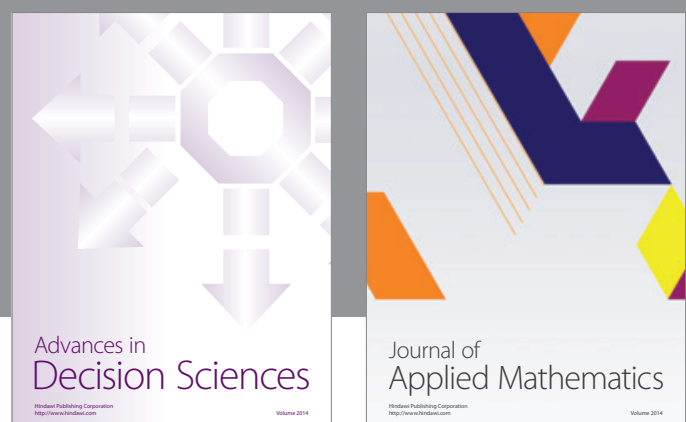

Journal of

Applied Mathematics
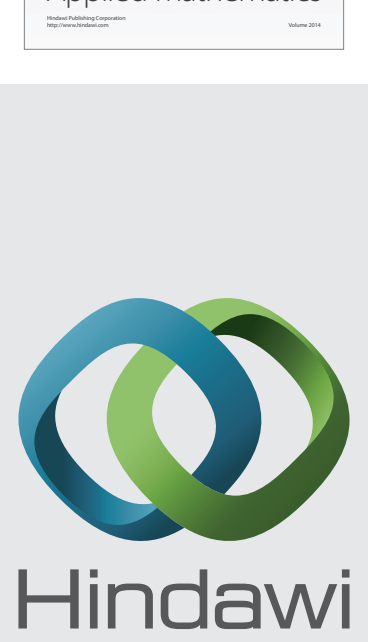

Submit your manuscripts at http://www.hindawi.com
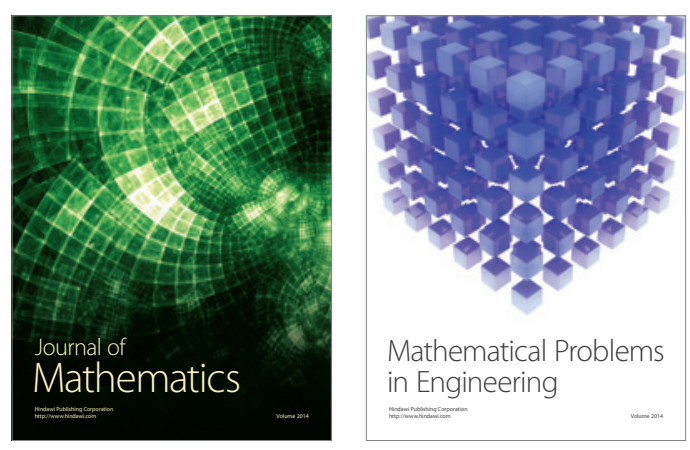

Mathematical Problems in Engineering
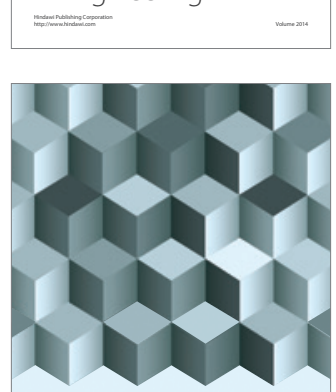

Journal of

Function Spaces
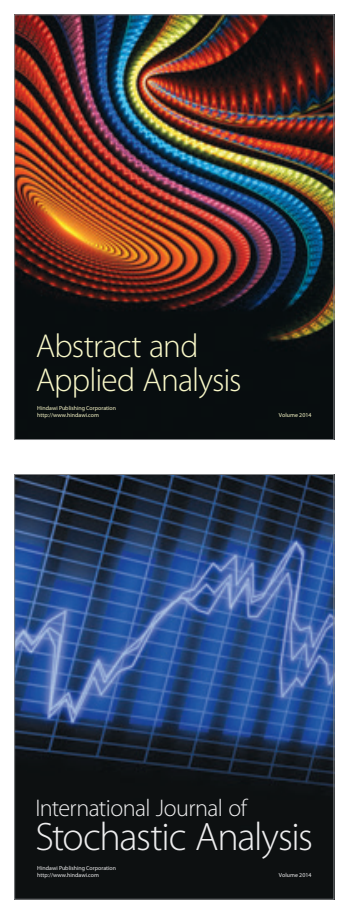

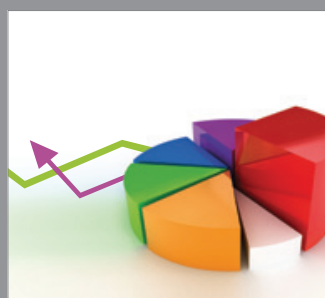

ournal of

Probability and Statistics

Promensencen
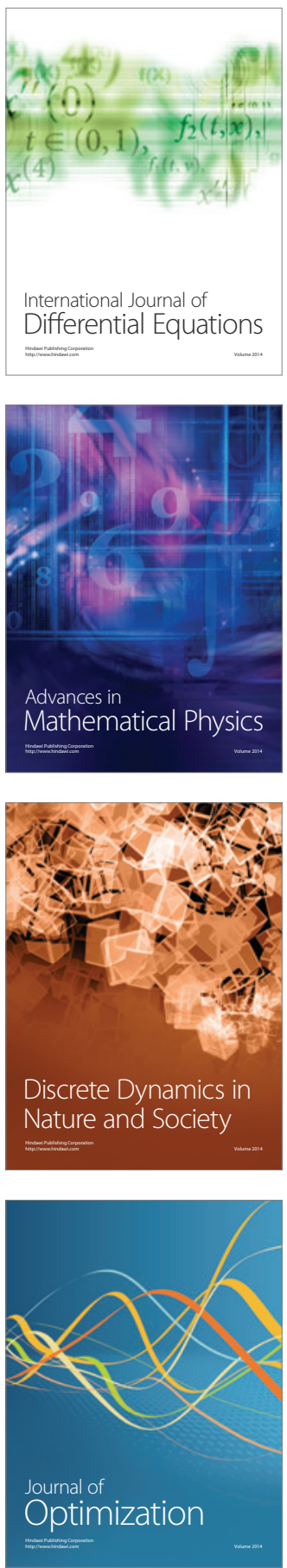